\title{
Design of Feedback in Interactive Multimedia Language Learning Environments
}

\author{
Vehbi Türel (Bingol)
}

\begin{abstract}
In interactive multimedia environments, different digital elements (i. e. video, audio, visuals, text, animations, graphics and glossary) can be combined and delivered on the same digital computer screen (TDM 1997: 151, CCED 1987, Brett 1998: 81, Stenton 1998: 11, Mangiafico 1996: 46). This also enables effectively provision and presentation of feedback in pedagogically more efficient ways, which meets not only the requirement of different teaching and learning theories, but also the needs of language learners who vary in their learning-style preferences (Robinson 1991: 156, Peter 1994: 157f.). This study aims to bring out the pedagogical and design principles that might help us to more effectively design and customise feedback in interactive multimedia language learning environments. While so doing, some examples of thought out and customized computerised feedback from an interactive multimedia language learning environment, which were designed and created by the author of this study and were also used for language learning purposes, will be shown.
\end{abstract}

\section{$1 \quad$ Literature review}

Not only can it be said that almost all researchers (Jordan/Mitchell 2009: 371-385, Lee 2008: 144-164, Bada/Okan 2000: 1-13) agree on the necessity and positive effects of constructive feedback, but it can also be said that language learners (LLs) prefer online feedback (Bridge/Appleyard 2008: 644:50). Although feedback is provided almost in all language learning materials, it is very often limited and inappropriate (Draper 2009: 306-315, Türel 2010: 399; 2000). Without any doubt, not only does inappropriate feedback not benefit LLs properly (Trinder 2002: 73, 80, 82), but such feedback is also not appreciated by LLs (ibid: 73, 82; Fleta et al. 1999: 52). The implication of this is that not only should feedback be provided in interactive multimedia language learning environments, but the feedback should also pedagogically be appropriate for the targeted learners and objectives.

Feedback, which is a reaction or response that is usually triggered and received by LLs and provided by teachers/materials writers (Ypsilandis 2002: 169), is generally categorised into two groups: (1) implicit/intrinsic feedback, which is a natural consequence of actions, and (2) explicit/extrinsic feedback, which happens as an external comment on natural actions (Peter 1994: 129; Laurillard 1993; Robinson 1989: 128). In interactive multimedia language learning environments, both implicit/intrinsic and explicit/extrinsic feedback need to be provided, as each has its own advantages. For example, explicit feedback provides the correct form and clearly indicates that the LLs' answer is wrong when their answer is incorrect. Such feedback helps LLs to be sure about what they have just done and focus on the part they are going to do next.

Feedback can be presented in different forms. (1) Feedback can be presented as explicit correction, which provides the correct form and clearly indicates that what LLs have said is incorrect. (2) Feedback can be presented as recasts. Recasts are utterances that repeat LLs' incorrect utterances, making only the changes necessary to produce correct utterances, without 
changing the meaning and saying explicitly that they are wrong. (3) Feedback can be presented as clarification requests, which provide phrases such as Pardon? and I don't understand. (4) Feedback can be presented as meta-linguistic clues, which supplies comments, information, or questions pertinent to the well-formedness of the LLs' utterances. (5) Feedback can be presented as elicitation, which directly elicits a reformulation from LLs by asking questions such as "How do we say this in English?" or by pausing to allow LLs to complete the utterance, or by asking LLs to reformulate their utterance. (6) Feedback can be presented in the form of repetition, which repeats the LLs' ill-formed utterance, highlighting the errors. (7) Feedback can be provided in the form of translation, which provides equivalents of TL in L1 (Nicholas et al. 2001: 757-58; Nagata 1997). There are, however, some questions that need to be answered. These are: (1) which forms of feedback do we need to provide in interactive multimedia language learning environments? (2) Which forms of feedback do LLs prefer? (3) Which forms of feedback under which conditions contributes to language learning more? These issues will be discussed below (Section 2 and 3 ).

LLs do not mind useful, positive and constructive feedback and conversely they use such feedback and find it useful (Jordan/Mitchell 2009: 371-385, Bridge/Appleyard 2008: 644:50, Lee 2008: 155, Bada/Okan 2000: 1-13, Gillespie/McKee 1999: 42, Kreindler 1998: 243-249, Peter 1994: 157-58, Dawey et al. 1995, Brandl 1995, Radecki/Swales 1988: 355-65). Feedback is mainly useful for conscious foreign language learning (FLL) (Jordan/Mitchell 2009: 308; Bitchener 2005: 193-203, Krashen 1984: 11), as (1) it facilitates the negotiation of meaning. (2) Feedback forces LLs to focus attention on certain aspects of the input (Smith 2003: 39-40, Williams 2001: 337, Chapelle 1997: 25f.) and output. (3) Feedback helps LLs improve meta-cognitive strategies (planning, monitoring and evaluating their comprehension and the effectiveness of their strategies) (and cognitive strategies) (Bacon 1992: 410, Ferney/Waller 2001: 154). All these are effective factors in FLL. The implication of these suggestions and results is that we, the material writers, need to provide positive and constructive feedback (1) to increase conscious FLL, (2) to draw LLs' attention to certain aspects of the input that is provided, and (3) to improve LLs' strategies. These can easily and effectively be implemented in interactive multimedia language learning environments, as explained below.

On condition there is appropriate feedback, (1) LLs can realise their errors, as certain types of feedback are more effective for certain types of errors (Lee 2004: 154). Errors happen consistently as a result of lack of knowledge. They are categorised into different groups in terms of separate aspects by different researchers. For example, Ellis (1997: 17, 19f.) categorises errors as: (a) universal errors, which are made by LLs from different backgrounds; (b) common errors, which are made by those who share the same L1 or linguistic family; (c) global errors, which violate the overall structure of a sentence; and (d) local errors, which affect only a single constituent in a sentence. Likewise, errors are also categorised as (a) discourse errors, (b) factual errors, (c) word errors and (d) syntactic errors (Lee 2004: 149-57). With appropriate feedback, (2) LLs can also realise their mistakes. Mistakes happen as LLs sometimes slip up, although LLs possess knowledge of the correct forms (ibid). With appropriate feedback (3) LLs can diagnose their problems and find out why they have failed. All these are likely to help LLs not to make the same mistakes and errors again and benefit from them (Peter 1994: 157f.). The implication for interactive multimedia language learning environments is that feedback should aim to help (1) LLs realise their mistakes and errors and (2) find out why they did wrong when their answers were incorrect.

Regarding the design of feedback in interactive multimedia environments, Peter (1994: 157f.) mentions that "positive program-feedback that allows learners to learn from their mistakes" as a feature that has "an impact on learning effectiveness". Moreover, Gillespie and McKee (1999: 40f.) indicate that "students need more reassurance when working on a computer than 
when completing pen and paper exercises. They want and need early feedback...". Therefore, the design of feedback in such environments is described as one of "two challenges facing software developers" under the hypothesis of ideal conditions for FLL (Clifford 1998: 5). The implication of these findings for interactive multimedia language learning environments is that feedback has to be (1) positive and constructive, (2) explanatory and (3) immediate.

As it is the case in the traditional materials, once feedback in interactive multimedia language learning environments are designed and created, the provided feedback cannot be amended. Necessary or needed changes in the provided feedback can only be made in newer version of the existing environments. This is a specific constraint of feedback in a multimedia learning environment.

All so far mentioned issues are "crucial factors for success or failure" of interactive multimedia language learning environments (Ypsilandis 2002: 172). When feedback is provided in such environments, where it can be in multiple format, forms and combined, interactive, simultaneous, customised, constructive, explanatory, intrinsic, extrinsic, and guide LLs to find out their mistakes and errors and help them to be conscious LLs and improve their strategies; then not only does feedback become more precise and valuable, but such feedback also requires more carefully thought out design (Robinson 1991: 156). Therefore, basing on the literature review and the author's so far pertinent experiences, for effective feedback design in interactive multimedia language learning environments, the following principles and guidelines are suggested from the point of view of pedagogy and design:

\section{Design of feedback from the point of view of pedagogy}

Feedback should be available in all cases for both correct and wrong answers. If the answers are correct, explanations should be given to indicate why the answers are correct. When the answers are wrong, the focus of feedback should be on the potential causes, not on errors. This is a requirement of the person perception theory, as it might cause LLs to develop negative judgements about their ability (Lee 2008: 158, Robinson 1991: 157), which is the last thing interactive multimedia environment writers would ever want to achieve. Instead, the feedback should guide LLs to overcome such difficulties on future occasions. Thus, the focus of the feedback should be on potential problems. If the potential failures are diagnosed and explained clearly and effectively or remedial actions taken, then LLs are more likely to benefit from them fully, as such feedback is more likely to help LLs to find out what and why they did wrong (Sheerin 1987: 129). Not only should the objective of such remedial actions be to help LLs overcome the present difficulties, but it should also help and guide LLs in the near future. When it is necessary interactive multimedia language learning environments should direct LLs to the specific parts of the software that can help overcome particular difficulties and misunderstanding. This can be done very easily through links. Such links can be syntax, pictures, dictionaries, instructions, cultural differences, tutorials and the like.

All types of feedback (i. e. explicit correction, recasts. clarification requests, meta-linguistic clues/descriptive, elicitation, repetition, translation (Eric/Lin 2007: 1122-1125, Nicholas et al. 2001: 757f.; Nagata 1997) need to be provided in multimedia learning environments, as each type of feedback can contribute to FLL in different ways. For example, the results of Eric/Lin's study (2007: 1123f.) reveal that while providing certain types of feedback (i. e. descriptive document, program debug and evaluation) enables the use of more advanced cognitive/meta-cognitive strategies, providing emotional wording feedback types reduce the use of cognitive/meta-cognitive strategies. In the same way, while factual errors require more clarification request feedback type, word errors require more repetition feedback type (Lee 2004: 154). In short, the right type of feedback that aims to achieve certain target objectives need to be provided with the certain types of language input for certain level of LLs. 
Feedback should be provided in the form of (1) global/general feedback and (2) local/specific feedback. The difference is that the former, which is also considered help/guidance, can be accessed from any part of the software (depending on the design of the software), while the latter (the feedback for both correct and incorrect answers) can be accessed only from the page/section where it is available. Providing feedback in the former format is appreciated and found useful by LLs, although the latter is preferred more (Turel 2004: 368f.). For example, in the interactive multimedia language learning environment, feedback was provided both in the form of global help (Figure 1) and local help (Figure 2).

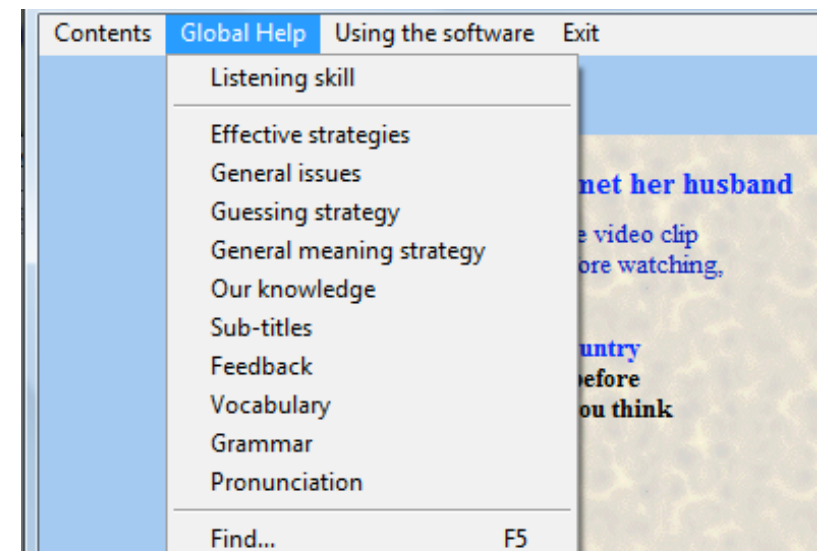

Figure 1: A sample of feedback in the form of 'global help'.

LLs can access the feedback in the form of global help from every single page in the interactive multimedia language learning environment by clicking any sub-menu (Figure 1). Such feedback can be more general and give advice about certain aspects of the input they use such as using effective strategies, general issues, the use of subtitles and feedback, vocabulary and grammar learning, picking up correct pronunciation, paying attention to stress, intonation, accents and other authentic features of the input.

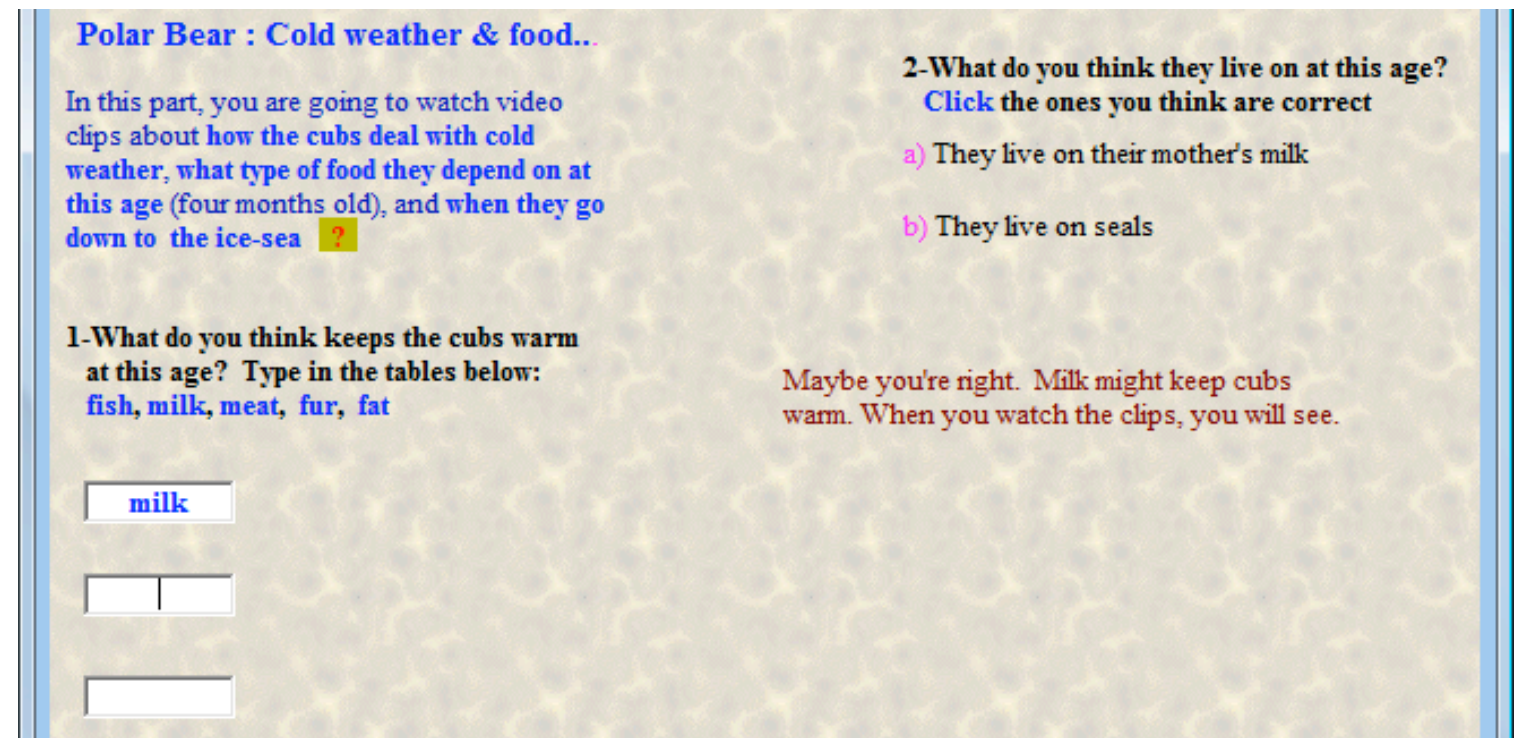

Figure 2: A sample of feedback in the form of local help.

When it comes to feedback in the form of local help, such feedback can only be available on a particular page where LLs work on. For example, in Figure 2, when LLs type the word milk as one of the answers at the pre-listening stage, they receive the feedback "Maybe you're right. Milk might keep cubs warm. When you watch the clips, you will see." This feedback is displayed on the bottom right section of the page (Figure 2). 
Feedback should not consist of only Yes/No or Correct/Incorrect. Some language software on market feature feedback which only consists of Yes/No or Correct/Incorrect. For example, English for Business - Introduction to a company, Beginning Kurmanji Kurdish, Advanced Turkish, Beginning Turkish (Turel 2011; 2010: 399; 2003: 592-602; 2000: 96). Although feedback in the mentioned software is pedagogically very satisfactory and beneficial; it does not feature any explanation of why an answer to a task is correct or wrong. The feedback only indicates whether an answer is correct or wrong and provides right answers when selected ones are wrong and this is really not enough (Nagata 1998: 24). LLs definitely need to be provided with more than this in many cases. In short, they should be told as much as possible, not only whether their answer is wrong or correct. Since feedback is useful for FLL in terms of different aspects (Jordan/Mitchell 2009: 308, Smith 2003: 39-40), as indicated above; feedback should also explain why an answer is correct or wrong and what strategies should be followed. In other words, feedback should help LLs to diagnose their problems and find out why they have failed. This is especially necessary for autonomous LLs as such LLs are by themselves. Therefore, interactive multimedia language learning environments, which are used as autonomous materials generally; need to function as a surrogate teacher so that they can raise LLs' consciousness and strategic knowledge.

Feedback should be immediate (Watts 1997: 4-5) because instant feedback can (1) have the effect of making linguistic input comprehensible, (2) correct on-going wrong interpretations when the selected answer is wrong, and (3) encourage LLs when the answer is correct. Providing instant feedback is also a requirement of the direct contrast hypothesis, which suggests that immediate feedback following errors is conducive to the perception of a contrast between errors and correct forms - a rejection of incorrect forms and their replacement by the correct forms (Nicholas et al. 2001: 721, Saxton 1997). Whether LLs access or read/view feedback immediately or not is something that they know and decide, although we need to encourage them to do so, and raise consciousness awareness about the role of instant access. For example, in Figure 3, as it is the case in all the provided figures in this article, feedback is immediate. As soon as LLs answer the question, they receive simultaneous feedback.

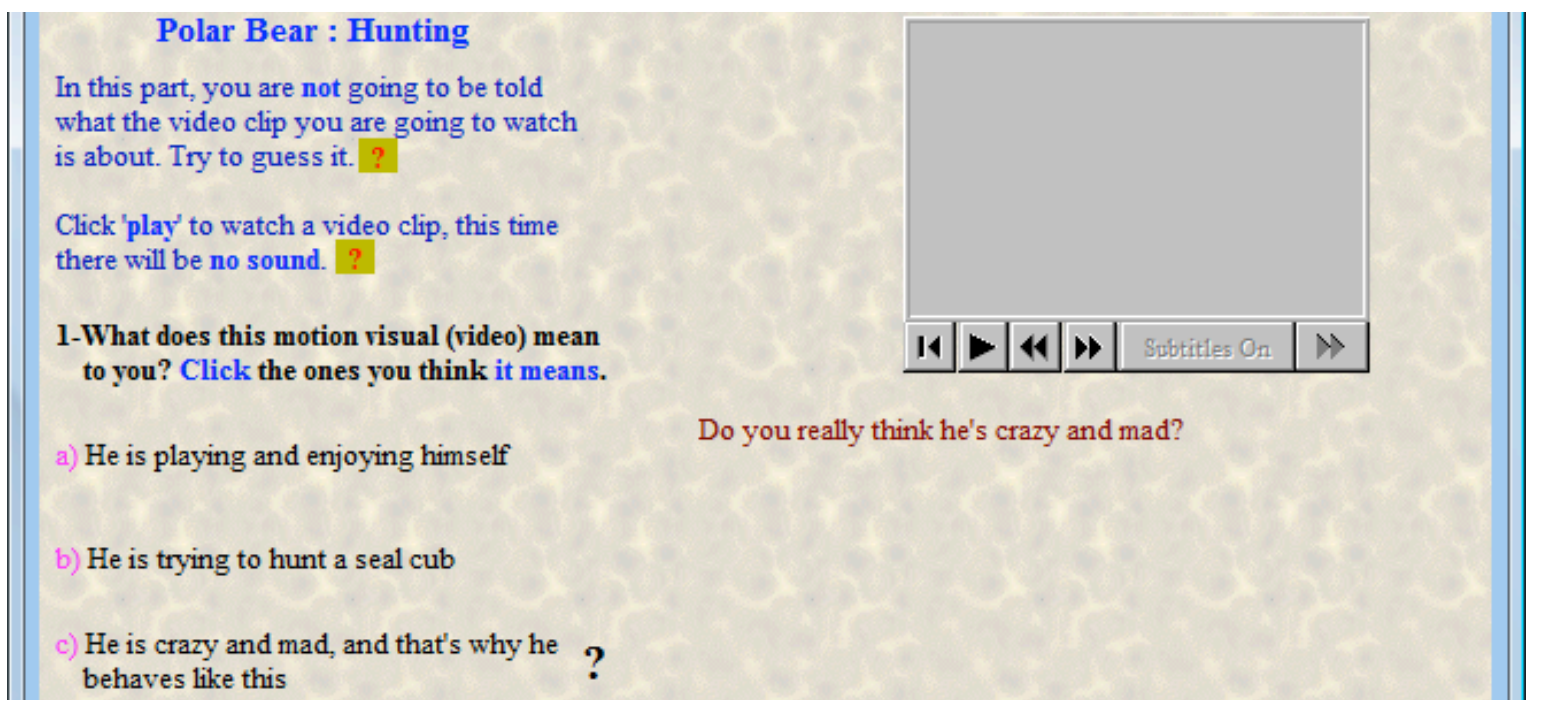

Figure 3: A sample of immediate feedback

In Figure 3, when the LLs click choice c) of Question 1 after they have watched the video, they receive immediate feedback, which says: "Do you really think he's crazy and mad" by additionally displaying a question mark (?) next to the choice. The objective of such immediate feedback is to make LLs think and correct their on-going wrong interpretations. When the answer is correct, then a tick $(\sqrt{ })$ is displayed next to the choice instead of displaying a question mark (?). 
Feedback, in some cases, should be conditional and customised. For example, in Figure 4, the LLs are requested to click the suitable choices (more than one: three choices). The feedback LLs receive change according to the choices they have made. In this way, the received feedback is customised according to the needs/choices of the target LLs.

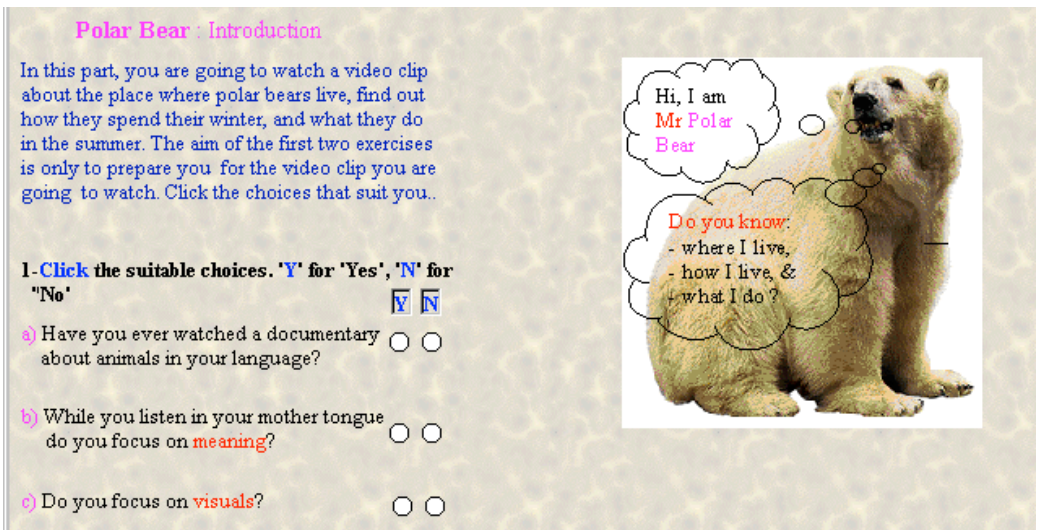

Figure 4: A sample task that is provided with conditional and customised feedback.

When LLs click "N" (No) choices for Question 1, they receive the feedback that is in the right side of Figure 5.

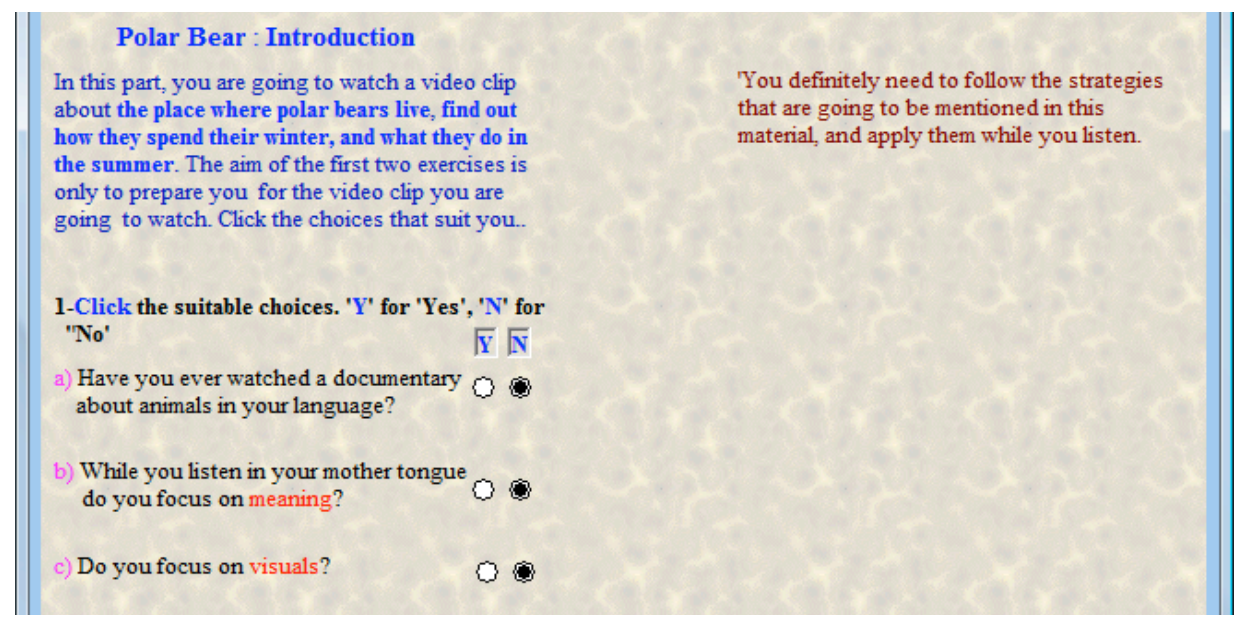

Figure 5: A sample task that is provided with conditional \& customised feedback.

When LLs click "Y" (Yes) choices for Question 1, they receive the feedback that is in the right side of Figure 6.

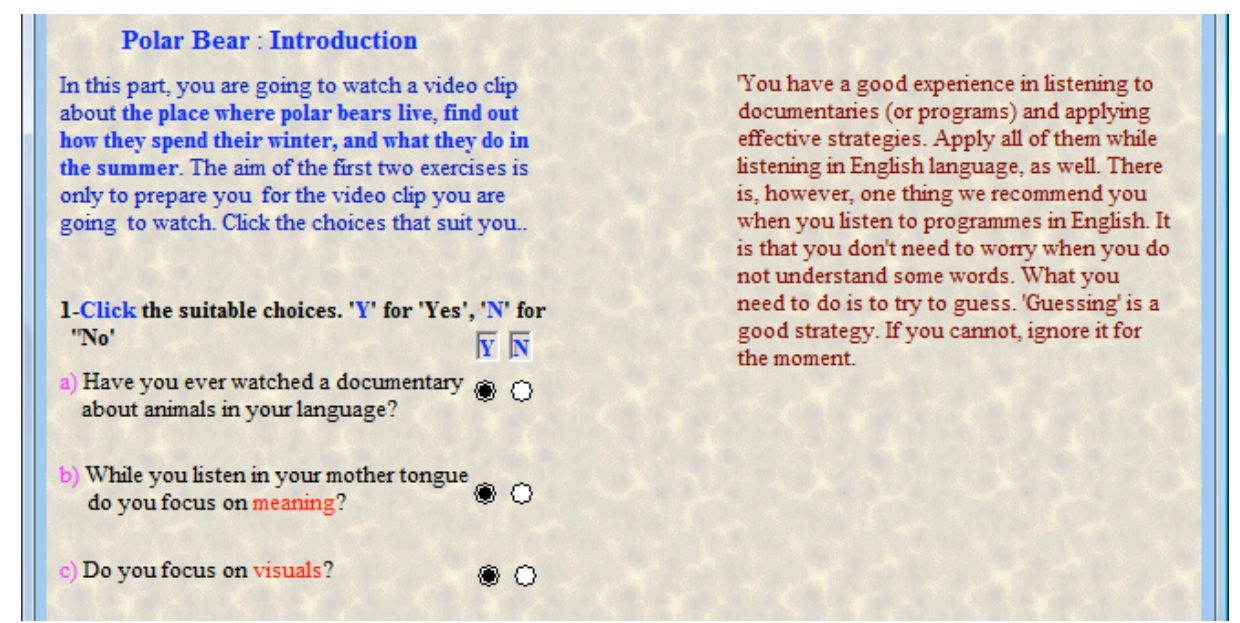

Figure 6: A sample task that is provided with conditional and customised feedback. 
When LLs click "Y" for choice a), "N" for choice b) and "Y" for choice c) of Question 1, then they receive the feedback that is in the right side of Figure 7. In short, feedback is customised and changes according to LLs' clicks or needs.

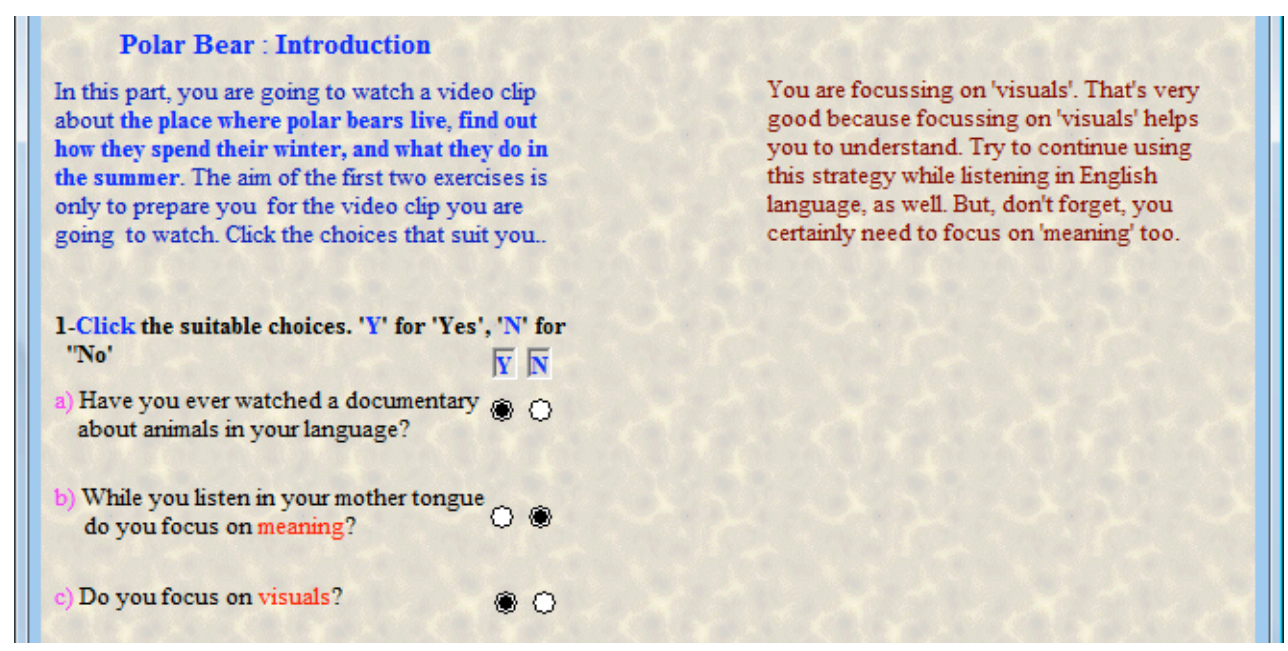

Figure 7: A sample task that is provided with conditional and customised feedback.

Feedback should sometimes be about the main key points that might help LLs to find out how to give their attention to such points (Figure 8 and 9) or use appropriate strategies to comprehend input.

Feedback should be simple, short, meaningful, communicative, accurate and comprehensible; focus on content (surface and deeper meaning, cultural aspects) and form (linguistic aspects) because only such feedback can create a learning situation. Unclear, too long, too much, complex and meaningless feedback cannot help at all (Davey et al. 1995: 33). For example, feedback in Figure 8 is clear and short.

Not only should feedback feature written texts, but it should also be multiple format feedback. Depending on the questions, the answers and the target LLs' needs, feedback can be visual oriented, aural oriented, textual oriented or optimum combinations (Figure 8), although multiple format feedback might not always be effective (Lima et al. 2005: 312f.). In short, the requirements of the dual-coding theory and the generative theory of multimedia (Ginther 2002: 133-167, Moreno/Mayer 2002, Al-Seghayer 2001: 220, Chun/Plass 1996: 515, Mayer/Sims 1994: 389-401, Paivio 1986) should be considered in the design of feedback. These theories suggest that LLs should be provided with more than one mode/element that targets to teach one thing and it is learnt better than those coded only in one mode because dual-code provides more paths of recall, which can aid to build two types of recall cues in memory. Likewise, the requirements of learning channels (Gyselinck et al. 2002: 665) and styles (Carson/Longhini 2002: 408, McLoughlin 1999: 222f., Brickell 1993:1f., Dunn 1983: 496-506, Reid 1987: 92) should also be considered in the design of feedback, as LLs vary in their learning channels and styles such as visual, auditory, kinaesthetic, tactile, group or individual learners and they can dominantly use either of them or a combination of them. While so doing, (the) cognitive overload (theory) should not be ignored (Kalyuga 2000: 162, Sweller 1999, Carroll 1977: 508). This theory, which "places a primary emphasis on working memory capacity limitations as a factor in instructional design", suggests that "too many elements of information may overwhelm working memory, decreasing the effectiveness of instruction"(Kalyuga 2000: 161, Sweller 1999, Hedberg et al. 1993: 6). 


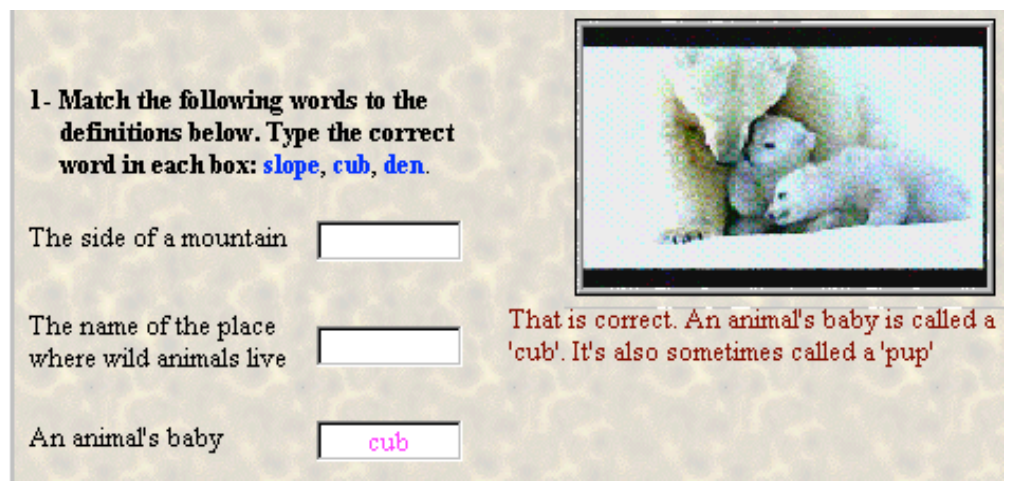

Figure 8: Sample feedback that consists of optimum combinations - text + video + audio

In Figure 8, when LLs type the word $c u b$ as a potential answer for "An animal's baby", not only "That is correct. An animal's baby is called a 'cub'. It is also sometimes called a 'pub'." is displayed as immediate feedback, but a video clip featuring a female bear and her two cubs also plays. Such a combination of feedback in interactive multimedia language learning environments is more likely to be effective for FLL.

Feedback should be provided in such a way that (1) LLs can find what they expect. (2) It should meet the needs of individual LLs who have different leaning style preferences (Radecki/Swales 1988: 355-365), as LLs approach feedback differently and the usefulness of feedback depends on the match between writers' intentions and LLs' expectations. However, it should not be forgotten that the concept of learning style seems to lack empirical support from cognitive psychology; students' subjective preferences may, of course, be different.

The requirements of the noticing hypothesis and the attention theory, which suggest that paying/drawing attention to (specific) forms in the input is necessary for learning new linguistic features of a language (Nicholas et al. 2001: 721; Williams 2001: 335; Leow 2001: 114; 144; Schmidt 1990: 141, 1993, 1995; Chapelle 1998: 23), should be considered in the design of feedback. For instance, LLs' attention can be drawn to salient features of input, which is an effective factor in FLL (Figure 9). Similarly, LLs' attention can be drawn to errors such as common, universal, global and local. This can raise consciousness awareness and strategic knowledge, which are important in FLL. It is a pity that most LLs are not aware of even common errors. While so doing, feedback should be explicit. For example, feedback in Figure 9, aims to draw LLs' attention to some parts of the input, which is "... but a female encumbered by cubs would be even less successful".

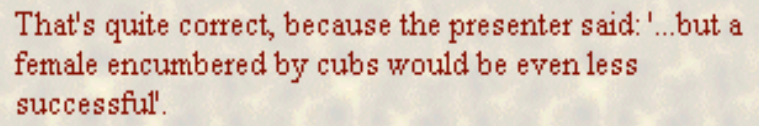

Figure 9: A sample feedback drawing attention to salient features of input

Feedback should, when needed, be detailed and informative enough. It should provide at least generic and specific information about student actions (Peter 1994: 129). Detailed and informative feedback should guide and direct LLs to develop cognitive and meta-cognitive strategies and function as an informal assessment procedure (Hurd et al. 2001: 346, Goh 2000: 72). This can also increase interactivity. For instance, LLs' attention can be drawn to elaboration + personal knowledge strategies, logic and general knowledge (Figure 10), resources, cultural differences (Figure 11 and 12), effective strategies (Figure 11) through feedback. 


\section{4- Click the statements that are NOT correct. \\ The mother is not protective \\ b) The cubs are allowed to wander off alone \\ A male bear can weigh around $600 \mathrm{Kg}$ \\ A) A female bear can weigh $600 \mathrm{Kg}$}

Well done. This statement is not correct. Like our own mothers, all animal mothers are protective. They do their best to protect their cubs. This is very natural.

Figure 10: Sample feedback drawing attention to analogy
We very often say where we are from when we introduce ourselves. Is it the same in your culture?

Figure 11: Sample feedback to draw attention to the similarities or differences between their and the target culture

As you've seen, knowing about 'British culture' helps you a lot to understand a listening text in English. For instance, it's very easy for somebody who is familiar with British weddings to understand such a topic.

Figure 12: Sample feedback to draw attention to the importance of being familiar with target culture in understanding target language

In Figure 10, LLs' attention is drawn to the similarities between our own mothers and a female bear as a mother (i. e. all animal mothers). In Figure 11, LLs' attention is drawn to the similarities and differences between their and the target culture. Similarly, in Figure 12, LLs' attention is drawn to the importance of being familiar with the target culture in understanding the target language. All these are pedagogically effective forms of feedback in interactive multimedia language learning environments.

For some cases, feedback might not be necessary. Instead, help can be provided. For such cases, help messages or advice can be provided (Ruhlmann 1995: 55-57; Figure 13).

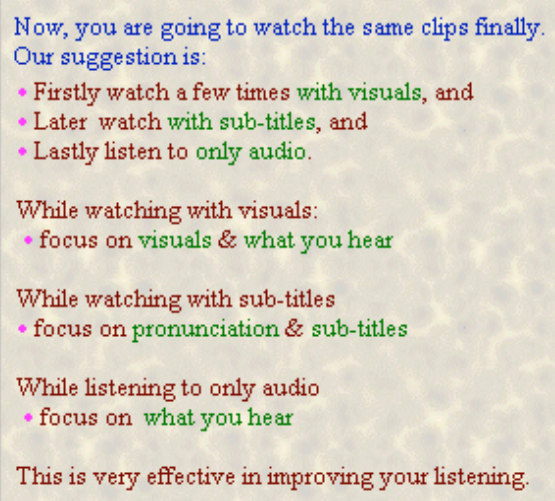

Figure 13: Sample feedback in the form of help/advice to draw LLs' attention to strategies and elements, and their role in understanding texts and improving listening.

In Figure 13, at the post listening stage, LLs are advised to watch the same audio clips first with visuals, later with sub-titles and lastly listen to only audio. They are also advised to focus on different aspects of the input in different form of watching or listening. For example, LLs are advised to focus on visuals while watching with visuals. LLs are advised to focus on pronunciation while watching with subtitles. Similarly, LLs are advised to focus on what they hear while they listen to only audio.

Feedback should sometimes be used to draw attention to the aspects LLs should have paid attention to and used to their advantage such as titles, cognates and other similar useful clues (Figure 14). 


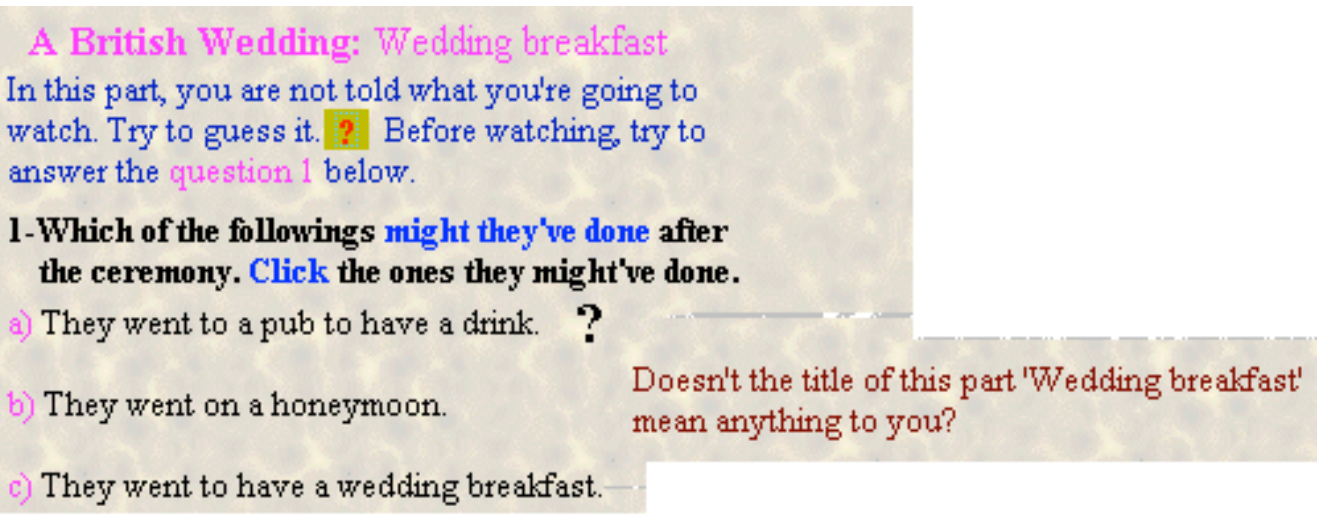

Figure 14: Feedback to draw attention to the aspects which LLs should have paid attention to

Feedback should sometimes give information to LLs about the assumptions underlying requesting them to answer particular questions. For example, feedback in Figure 15, which is displayed below the video stage, indicates that the objective of Question 1 of Polar Bear: Hunting lesson is to draw their attention to the role of visuals in understanding what they watch.

\section{Polar Bear : Hunting}

In this part, you are not going to be told what the video clip you are going to watch is about. Try to guess it. ?

Click 'play' to watch a video clip, this time there will be no sound. ?

1-What does this motion visual (video) mean to you? Click the ones you think it means.

a) He is playing and enjoying himself

b) He is trying to hunt a seal cub

c) He is crazy and mad, and that's why he behaves like this

Figure 15: Feedback to draw attention to the assumptions underlying certain questions

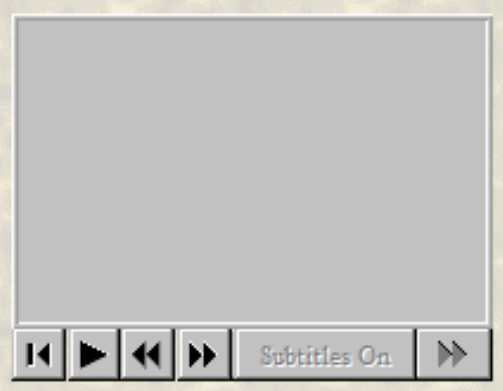

Here, the aim is to draw your attention to the role that visuals have in helping us understand what is said

Last but not least, feedback should not be used for testing, but only draw out personal involvement (Kreindler (1988:243-249).

\section{Design of feedback from the point of view of design}

Feedback in interactive multimedia language learning environments should be crystal clearly and consistently displayed (Ferney/Waller 2001: 154, Hemard 1997: 2-27, Watts 1997: 7). There should be consistency in place, size, font, colour, amount and so on as much as possible. This is especially vital for autonomous materials since such LLs are by themselves. Simple, clear and consistent feedback in terms of place, size, font, colour and amount can help enable LLs to take control of their learning and available feedback effectively. Moreover, such feedback can give interactive multimedia language learning environments a professional look and make working with the software enjoyable. Otherwise, since a wide range of elements can be combined and presented in the same interactive multimedia language learning environment, presenting feedback in different font sizes and types, colours and positions can easily make the screen look confusing and complex. For example, in the above figures, which were designed and developed by the author and thus copyright on these figures or screenshots 
belong to him, feedback was mainly and consistently displayed at the bottom right side of the page in the same font size, font type and colour (Figure 15).

To draw LLs' attention and to provide user friendly interactive multimedia language learning environments, feedback fields in different consistent colours as well as forms can be used for right and wrong answers (Ruhlmann 1995: 55). This was an aspect that the author of this study could not think of during the design and creation of his first interactive multimedia language learning environment.

LLs' attention can be drawn to certain aspects in other ways, as well. For example, likewise, in Peter's study (1994: 131) an orchestral chord is played if students give a true respond otherwise a duck quack sound is played. Similarly, different sounds can be used. Say, clapping sound can be played if LLs answer correctly and a chicking sound can be played if LLs respond incorrectly. Here, of course, consistency is a must. To this end, the author of this study used different consistent animations with feedback for the wrong and right answers in some parts of his first interactive multimedia language learning environment.

Alternatively, instead of sound, (sometimes) verbose praise can be used. Here we need to be very careful because praises for true answers andsounds can quickly become tiresome, (Hoffman 1995-6: 26). According to the experiences of the author of this study, when LLs are children verbose praises should be always used. Praises should be different as much as possible such as "well done", "that's right", "that's correct", '"hat's excellent", "that's ok", "alright", "good child", "good", "great", "you're right", "good work", "right", "excellent", "yes", "you're great", "brilliant", "perfect", "perfectly well-done", and so on. Such a wide range of variety can avoid potential tiresome. Alternatively, praise can be made in different ways. For example, in his study Peter (1994: 128) rewards the students with an animated firework display and some spinning test saying 'Well done' when they have completed a task correctly. When LLs are adult, such praises do not need to be used at all. Instead, reasonable and logical explanations can be more effective.

Moreover, while providing feedback, the LLs can be referred to with their own names. Technically, this can be programmed in interactive multimedia language learning environments. To this end, the author of this study, for instance, provided feedback with the LLs' actual names such as: "Well done, Mary. Your answer is correct, but you should also try to pay attention to the visuals". LLs seemed to have enjoyed such a privilege and were also surprised to find out that they could be referred to with their own respected names.

Feedback needs to be interactive. This can be achieved by providing instant and explanatory feedback, presenting different combined media types as feedback (Figure 8 above), and focussing and integrating cultural differences in feedback (Figure 11 and 12 above).

Design of feedback in interactive multimedia language learning environments should not hinder LLs accessing or skipping any feedback, link or part when they need or want. LLs should be able to work by following their preferred learning style and pace. In particular, when LLs review, they want, to a greater extent, to skip certain types of feedback, as they are already familiar with, and thus they will not need to re-read or re-view each time they review. In other words, LLs' progress should not be delayed until they have accessed the provided feedback.

\section{Conclusion}

The power of interactive multimedia language learning environments, where different digital elements (i.e. video, audio, visuals, text, animations, graphics, glossary and so on) can be combined and delivered on the same digital computer screen, enables us to provide and present more effective, customised and immediate feedback for FLL. Taking the above presented design principles and guidelines that need to be considered in providing and presenting feed- 
back in interactive multimedia language learning environments can further enable us to design and create as a whole more effective language learning materials.

\section{References}

Al-Seghayer, Khalid (2001): "The effect of multimedia annotation modes on L2 vocabulary acquisition: a comparative study". Language Learning \& Technology 5/4: 202-232.

Bacon, Susan M. (1992): "Authentic Listening in Spanish: How Learners Adjust Their Strategies to the Difficulty of the Input". Hispania 75/2: 398-411.

Bada, Erdogan/Okan, Zuahl (2000): "Students' language learning preferences". TESL-EJ 4/3: $1-13$.

Bitchener, John/Young, Stuart/Cameron, Denise (2005): "The effect of different types of corrective feedback on ESL student writing". Journal of Second Language Writing 14: 191-205.

Brandl, Klaus K. (1995): "Strong and Weak Students' Preferences for Error Feedback Options and Responses". The Modern Language Journal 79: 194-211.

Brett, Paul (1998). "An intuitive, theoretical and empirical perspective on the effectiveness question for multimedia". In: Cameron, Keith (ed.): Multimedia CALL: Theory and Practice. Exeter, Elm Bank Publication: 81-92.

Brickell, Gwyn (1993): "Navigation and learning style". Australian Journal of Educational Technology 9/2: 103-114.

Bridge, Pete/Appleyard, Rob (2008): "A comparison of electronic and paper-based assignment submission and feedback". British Journal of Educational Technology 39/4: 644650.

Carroll, John B. (1977): "On learning from being told". In: Wittrock, Merlin C. (ed.): Learning and Instruction. Berkeley, CA: McCutchan: 496-512.

Carson, Joan G./Longhini Ana (2002): "Focussing on learning styles and strategies: A diary study in an immersion setting". Language Learning 52/2: 401-438.

CCED (Collins Cobuild Educational Dictionary) (1987). London: HarperCollins Publishers.

Chapelle, Carol A. (1997): "CALL in the Year 2000: Still in Search of Research Paradigms?". Language Learning \& Technology 3/1: 19-43.

Chapelle, Carol A. (1998): "Multimedia CALL: Lessons to be learned from Research on Instructed SLA". Language Learning \& Technology 2/1: 22-34.

Chun, Dorothy/Plass, Jan (1996): "Facilitating Reading Comprehension with Multimedia". System 24/4: 503-519.

Clifford, Ray (1998): "Mirror, mirror, on the Wall: reflections on computer assisted language learning". CALICO Journal 16/1: 1-11.

Davey, Denise/Jones, Kirsten G./Fox, Jeremy (1995): "Multimedia for language learning: Some course design issues". Computer Assisted Language Learning 8/1: 31-44.

Draper, Stephen W. (2009): "What are learners actually regulating when given feedback?". British Journal of Educational Technology 40/2: 306-15.

Dunn, Rita (1983): "Learning style and its relation to exceptionality at both ends of the spectrum". Exceptional Children 49: 496-506.

Eric ZhiFen Liu/Lin, Sunny S. J. (2007): "Relationship between peer feedback, cognitive and metacognitive strategies and achievement in networked peer assessment". British Journal of Educational Technology 38/6: 1122-25.

Ferney, Derrik/Waller, Sharon (2001): "Reflections on multimedia design criteria for the international language learning community". Computer Assisted Language learning 14/2: $145-168$.

Fleta, Begoña M. et al. (1999): "Evaluating multimedia programs for language learning: a case study". ReCALL 11/3: 50-57. 
Gillespie, John/McKee, Jane (1999): "Resistance to CALL: degrees of student reluctance to use CALL and ICT". ReCALL 11/1: 38-46.

Ginther, April (2002): "Context and Content Visuals and Performance on Listening Comprehension Stimuli." Language Testing 19/2: 133-167.

Goh, Christine C. M. (2000): "A Cognitive perspective on language learners' listening comprehension problems". System 28: 55-75.

Gyselinck, Valérie et al. (2002): "Visuospatial Memory and Phonological Loop in Learning from Multimedia". Applied Cognitive Psychology 16: 665-685.

Hedberg, John. G./Harper, Barry/Brown, Christine (1993): "Reducing cognitive load in multimedia navigation". Australian Journal of Educational Technology 9/2: 157-181.

Hémard, Dominique P. (1997). "Design Principles and Guidelines for Authoring Hypermedia Language Learning Applications". System 25/1: 9-27.

Hoffman, Suzanne (1995-1996): "Computers and Instructional Design in Foreign Language/ESL Instruction". TESOL JOURNAL Winter: 24-29.

Hurd, Stella/Beaven, Tita/Ortega, Ana (2001): "Developing autonomy in a distance language learning context: issues and dilemmas for course writers". System 29: 341-355.

Jordan, Sally/Mitchell, Tom (2009): "e-Assessment for learning? The potential of short-answer free-text questions with tailored feedback". British Journal of Educational Technology 40/2: 371-385.

Kalyuga, Slava (2000): "When using sound with a text or picture is not beneficial for learning". Australian Journal of Educational Technology 16/2: 161-172.

Krashen, Stephen D. (1984): Principles and Practice in Second Language Acquisition. Oxford: Pergamon.

Kreindler, Isabelle (1988): "Designing feedback that is hard to ignore: A boost from multimedia". In: Cameron, Keith (ed.): Multimedia CALL: Theory and Practice. Exeter, Elm Bank Publication: 243-249.

Laurillard, Diana (1993): Rethinking University Teaching: A framework for the Effective Use of Educational Technology. Routledge: London.

Lee, Icy (2008): "Student reactions to teacher feedback in two Hong Kong secondary classrooms". Journal of Second Language Writing 17: 144-164.

Lee, On-Soon (2004): "Error types and feedback of NNS-NNS interactions". http://www.paaljapan.org/resources/proceedings//PAAL9/pdf/LeeOnso.pdf, accessed July $17,2012$.

Leow, Ronald P. (2001): "Attention, awareness, and foreign language behaviour". Language Learning 51, Issue Supplement 1: 113-155.

Lim, Kai. H./O'Connor, Marcus J./Remus, William E. (2005): "The impact of presentation media on decision making: does multimedia improve the effectiveness of feedback?" Information \& Management 42: 305-316.

Mangiafico, Lara F. (1996): The Relative Effects of Classroom Demonstration and Individual Use of Interactive Multimedia on Second Listening Comprehension. Unpublished Ph.D. Thesis, Vanderbilt University: Faculty of Graduate School.

Mayer, Richard E./Sims, Valerie K. (1994): "For whom is a picture worth a thousand words? Extension of a dual coding theory of multimedia learning". Journal of Educational Psychology 86/3: 389-401.

McLoughlin, Catherine (1999): "The implications of the research literature on learning styles for the design of instructional material". Australian Journal of Educational Technology 15/3: 222-241.

Moreno, Roxana/Mayer, Richard E. (2002): "Verbal redundancy in Multimedia Learning: When reading helps listening". Journal of Educational Psychology 94/1: 156-163.

Nagata, Noriko (1997): "The effectiveness of computer-assisted metalinguistic instruction: A case study in Japanese". Foreign Language Annals 30/2: 20-34. 
Nagata, Noriko (1998): "Input vs. Output Practice in Educational Software for Second Language Acquisition". Language Learning \& Technology 1/2: 23-40.

Nicholas, Howard/Lightbown, Patsy/Spada, Nina (2001): "Recasts as Feedback to Language Learners". Language Learning 51/4: 719-758.

Paivio, Allan (1986): Mental representations: A dual coding approach. New York: OUP.

Peter, Matthew (1994): Investigating into the Design of Educational Multimedia: Video, Interactivity and Narrative. Unpublished Ph.D. thesis, Open University, No: DX185077.

Radecki, Patricia M./Swales, John (1988): "ESL Student Reaction to Written Comments on their Written Work". System 16: 355-365.

Reid, Joy M. (1987): "The Learning Style Preferences of ESL Students". TESOL Quarterly 21/1: 87-111.

Robinson, Gail L. (1989): "The CLCCS CALL Study: Methods, Error Feedback, Attitudes, and Achievement". In: Flint Smith, William M. (ed.): Modern Technology in FLE: Applications and Project. Lincolnwood, IL: National Textbook Co.: 119-133.

Robinson, Gail L. (1991): "Effective feedback strategies in CALL: Learning theory and empirical research". In: Dunkel, Patricia (ed.): Computer-Assisted Language Learning and Testing: Research Issues and Practice. New York: Newbury House: 155-167.

Ruhlmann, Felicitas (1995): "Towards Replacement of the Teaching Process: The Emulation of the Teaching Process with CAL and its Implications for the Design of A Multimedia CAL Tutorial". Computer Assisted Language Learning 8/1: 45-61.

Saxton, Matthew (1997): "The contrast theory of negative input". Journal of Child Language 24/1: 139-161.

Schmidt, Richard W. (1990): "The role of consciousness in second language learning". Applied Linguistics 11/2: 129-158.

Schmidt, Richard W. (1993): "Awareness and second language acquisition". Annual Review of Applied Linguistics 13: 206-226.

Schmidt, Richard. W. (1995): "Consciousness and foreign language learning: A tutorial on the role of attention and awareness in learning". In: Schmidt, Richard W. (ed.): Attention and Awareness in Foreign Language Learning (Technical Report No. 9). Honolulu, HI: University of Hawai'i, Second Language Teaching and Curriculum Center: 1-63.

Sheerin, Susan (1987): "Listening comprehension: Teaching or testing?". ELT Journal 41/2: 126-131.

Smith, Bryan (2003): "Computer-mediated negotiated interaction: An expanded model". The Modern Language Journal 87: 38-57.

Stenton, Tony (1998): "Hypermedia: a new consensus for the 1990's". In: Cameron, Keith (ed.): Multimedia CALL: Theory and Practice. Exeter, Elm Bank Publications: 11-16.

Sweller, John (1999): Instructional Design in technical areas. Melbourne: ACER Press.

TDM (The Dictionary of Multimedia): (1997). Teddington: Peter Collin Publishing.

Trinder, Ruth (2002): "Forum: Multimedia in the Business English Classroom: The learners' point of view". Computer Assisted Language Learning 15/1: 69-84.

Türel, Vehbi (2000): "Talk Now! Learning Turkish". CALICO Journal 18/1: 91-100.

Türel, Vehbi (2003): "Beginning Turkish". CALICO Journal 20/3: 592-602.

Türel, Vehbi (2004): Design of Multimedia Software: Investigating the Design of Some Elements of Interactive Multimedia Listening Software for Autonomous Intermediate Language Learners. Unpublished Ph.D. Thesis, The University of Manchester, UK.

Türel, Vehbi (2010): "Advanced Turkish". ReCALL 22/3: 396-401.

Türel, Vehbi (2011): "Beginning Kurmanji Kurdish". CALICO Journal 28/3: 766-776.

Watts, Noel (1997): "A learner-based design model for interactive multimedia language learning packages". System 25/1: 1-8.

Williams, Jessica (2001): "The effectiveness of spontaneous attention to form". System 29: $325-340$. 
Vehbi Türel: Design of Feedback in Interactive Multimedia Language Learning Environments 49

Ypsilandis, G. S. (2002): "Feedback in Distance Education". Computer Assisted Language Learning 15/2: 167-181. 